\title{
Measuring Human Queues Using WiFi Signals
}

\author{
Yan Wang \\ Stevens Institute of \\ Technology \\ Hoboken, NJ 07030, USA \\ ywang48@stevens.edu \\ Yingying Chen \\ Stevens Institute of \\ Technology \\ Hoboken, NJ 07030, USA \\ yingying.chen@stevens.edu
}

\author{
Jie Yang \\ Oakland University \\ Rochester, MI 48309, USA \\ yang@oakland.edu
}
Marco Gruteser
Rutgers University
North Brunswick, NJ 08902, USA
gruteser@rutgers.edu

\author{
Hongbo Liu \\ Stevens Institute of \\ Technology \\ Hoboken, NJ 07030, USA \\ hliu3@stevens.edu \\ Richard P. Martin \\ Rutgers University \\ North Brunswick, NJ 08902 , \\ USA \\ rmartin@rutgers.edu
}

\begin{abstract}
We investigate using smartphone WiFi signals to track human queues, which are common in many business areas such as retail stores, airports, and theme parks. Real-time monitoring of such queues would enable a wealth of new applications, such as bottleneck analysis, shift assignments, and dynamic workflow scheduling. We take a minimum infrastructure approach and thus utilize a single monitor placed close to the service area along with transmitting phones. Our strategy extracts unique features embedded in the signal traces to infer the critical time points when a person reaches the head of the queue and finishes service, and from these inferences we derive a person's waiting and service times. We develop a feature driven approach in our system. Extensive experiments conducted both in the laboratory demonstrate that our system is robust to queues with different waiting time. We show that in spite of noisy signal readings, our methods can measure important time periods in queue (e.g., service and waiting times) to within a 10 second resolution.
\end{abstract}

\section{Categories and Subject Descriptors}

H.4.m [Information Systems Applications]: [miscellaneous]

\section{General Terms}

Measurement, Experimentation

\section{Keywords}

Human Queue, WiFi, Smartphone

\section{INTRODUCTION}

The popular usage of smartphones and their data-intensive apps has created novel opportunities to exploit such network traffic for monitoring and optimizing real-world processes. Research has shown, for example, how cellular call data records can be used to infer large

Permission to make digital or hard copies of part or all of this work for personal or classroom use is granted without fee provided that copies are not made or distributed for profit or commercial advantage, and that copies bear this notice and the full citation on the first page. Copyrights for third-party components of this work must be honored. For all other uses, contact the owner/author(s). Copyright is held by the author/owner(s)

MobiCom'13, September 30-October 4, 2013, Miami, FL, USA

ACM 978-1-4503-1999-7/13/09.

http://dx.doi.org/10.1145/2500423.2504584.

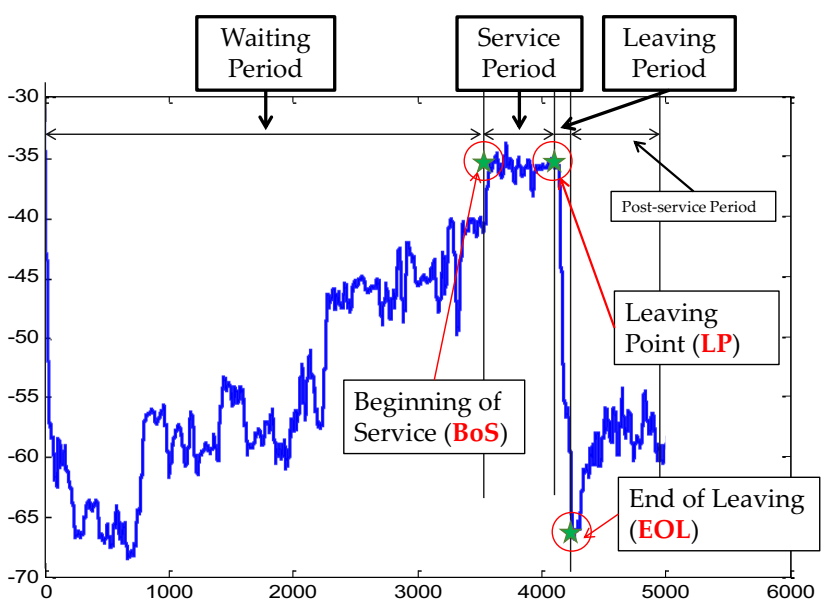

Figure 1: Illustration of special queue-related patterns embedded in the RSS trace collected from a smartphone in a human queue.

scale transportation patterns (e.g., [1]) or how cellular signal traces allow inferring the level of congestion on roadways [2]. In this work, we ask whether signal power readings from phone traffic are also sufficient, to monitor a much finer-scale, yet common, process: human queues.

Such queues are a familiar and often frustrating occurrence, for example in retail stores, banks, theme parks, hospitals and transportation stations. Note that we interpret the concept of a queue loosely, people do not need to stand in line but could sit in a waiting room and do not always need to be served in a strict first in, first out order. Real-time quantification of the waiting times and service times in such queues, allows optimizing service processes across ranging from retail, to heath care, to transportation and entertainment. For example, many hospital emergency departments surveys have average waiting times of several hours [3]. More complete wait and service time statistics allows customers, travelers, managers and service providers make changes to their behavior and processes. For example, an airport checkpoint might be experiencing abnormal delays and require intervention by diverting screeners from queues with shorter waiting times. Customers also can benefit, for example, knowing at what times retail store checkout lines can be expected to be shorter. 


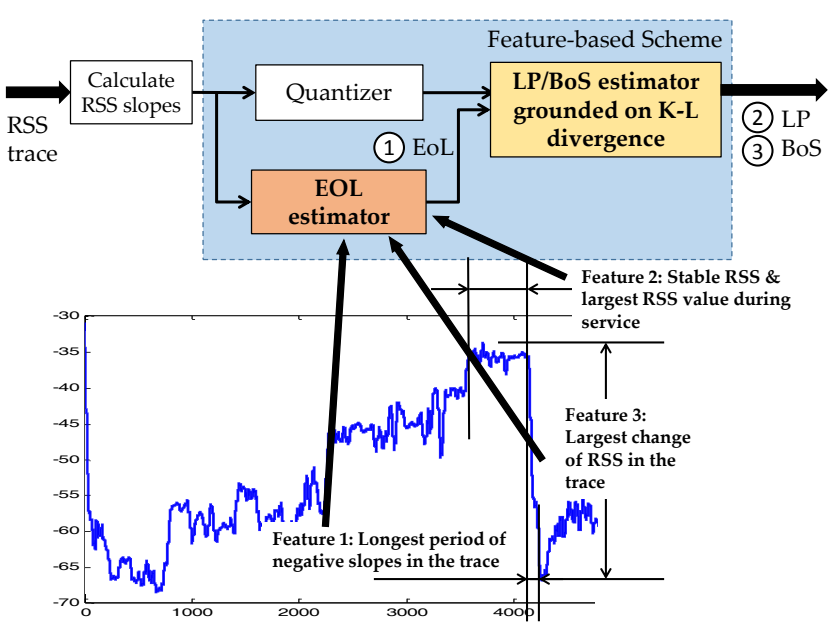

Figure 2: Flow of feature-driven scheme to find EoL, LP, and BoS in a time-reversed way.

Existing solutions to the queue monitoring problem often rely on cameras [4] or special sensors (e.g., infrared [5] or floor mats [6]) at multiple locations. Bluetooth signals emanating from phones have also been used to measure travel times between two sensing points, both at airports [7] and for vehicle traffic [8]. All these solutions require multiple sensors to fully monitor a single queue, which increases installation and system cost. In addition, prior techniques using wireless networks were too coarse-grained to differentiate between the waiting time and service time and vision techniques face increased privacy issues.

Our approach uses only a single sensor, a WiFi monitor near the head of the queue that measures the received signal strength of packets emitted from phones. Intuitively, the received signal power should follow a known pattern. As the person moves towards the service point, the phone moves closer to the monitor and the received signal power should increase. When the person is receiving service, the signal power should be very strong and relatively constant. Finally, when the person exits the service point, there should be a rapid decline in signal strength. For example, Figure 1 presents the RSS trace of a smartphone in a queue collected from a single WiFi monitor at the service desk in a coffee shop. We observe that the captured RSS trace reflects the patten of the distance between the person carrying the phone to the service desk. In particular, the RSS exhibits the following unique patterns: (1) the RSS has a slowly increasing trend during the waiting period; (2) it then becomes stable at high RSS values when the person carrying the phone is in the service period; and (3) it finally drops quickly when the person is leaving the service desk after got service in his leaving period.

Accurately discerning the points where the person begins and ends service is challenging, however, in this single-point monitoring system, because the multi-path, shadowing, and fading components of a signal are quite dynamic due to the movement of many people. We therefore investigate a direct feature-driven approaches for extracting the waiting and service times from a signal trace. We experimentally evaluate our approach in a laboratory with 90 traces. We find our approach is robust and able to measure the waiting time to with average errors less than $10 \mathrm{~s}$.

\section{FEATURE-DRIVEN QUEUE PARAMETER DETERMINATION}

We find that the important periods of a human queue are separated by three critical time points, namely Beginning of Service
(BoS), Leaving Point (LP), and End of Leaving (EoL). By deducing these three time points, our system is able to track the important queue time periods including waiting, service, and leaving periods.

Toward this end, we develop a feature-driven scheme in our system to identify the critical time points in a human queue. The feature-driven approach utilizes the unique features extracted from RSS traces to identify these time points. We next describe the details of this approach.

When designing the feature-driven scheme, we consider directly applying the features associated with the leaving period in the RSS trace to determine the EoL, LP, and BoS in a time-reversed manner. Because the RSS value changes dramatically when people leave the queue after service, the features associated with the leaving period are the most obvious and easy to be extracted. In particular, we identify three features extracted from the RSS trace associated with the leaving period:

1. The leaving period has the longest consecutive negative-slope segments of the selected RSS trace.

2. The RSS values before the leaving period are stable with the highest amplitude of the selected RSS trace.

3. The leaving period experiences the largest decreasing of RSS in the selected RSS trace.

The feature-driven scheme consists of three components: EoL Estimator, Quantizer, and $L P / B o S$ Estimator. We illustrate the flow of the feature-driven scheme in Figure 2.

EoL Estimator. Assume there is a group of $M$ segments with consecutive negative slopes in the RSS trace $\mathbb{G}=\left\{G_{1}, \ldots, G_{M}\right\}$, and each segment $G_{i}$ lasts for a period of $T_{i}=t_{1}^{i}-t_{0}^{i}$, where $t_{1}^{i}$ and $t_{0}^{i}$ denote the starting time and the ending time of that segment, respectively. The EoL estimator determines the time $t_{0}^{i}$ to be $\mathrm{EoL}$ when a segment $G_{i}$ is most likely to be a leaving period. To determine the likelihood of being a leaving period, we first define the significance of the three features described above as: 1) $\frac{T_{i}}{\max (\mathbb{T})}$, where $\mathbb{T}$ denotes time length of all the segments in $\mathbb{G} ; 2) \frac{S_{i}}{\max (\mathbb{S})}$, where $S_{i}$ is the average RSS over a time window $L$ before $t_{1}^{i}$, and $\mathbb{S}$ denotes all such average RSS; 3$) \frac{R_{i}}{\max (\mathbb{R})}$, where $R_{i}$ is the ratio of the RSS at $t_{0}^{i}$ and $t_{1}^{i}$, and $\mathbb{R}$ denotes all such RSS ratio, respectively. We further define an utility function which is a weighted sum of the significance of the three features:

$$
u_{i}=\alpha \cdot \frac{T_{i}}{\max (\mathbb{T})}+\beta \cdot \frac{S_{i}}{\max (\mathbb{S})}+\gamma \cdot \frac{R_{i}}{\max (\mathbb{R})},
$$

where $\alpha, \beta$, and $\gamma$ are the weights. The leaving period thus can be determined as the segment $G_{i}$ that maximizes the utility function $u_{i}$. And the time $t_{0}^{i}$ of such a segment $G_{i}$ is then declared as the EoL. To determine the weights in Equation (1), we use a heuristic approach by counting the occurrence of each feature in a small portion of the collected traces (e.g., 20 traces) and respectively using the ratio of each feature's occurrence to the number of traces as their weights. For example, if feature 1 has been found true for 10 times in 20 traces, the weight for feature 1 is then 0.5. Based on our experiments, we empirically find that when $\alpha=0.4, \beta=0.8$ and $\gamma=0.8$, the unique features can be extracted from the RSS trace accurately.

Meanwhile, we compute the slopes of each RSS sample in the selected RSS trace. A Quantizer performs normalization and quantization on the computed RSS slopes.

LP/BoS Estimator. Our feature-driven scheme then takes the input from the EoL Estimator together with the quantized RSS slopes to identify the leaving point (LP) and beginning of service point 


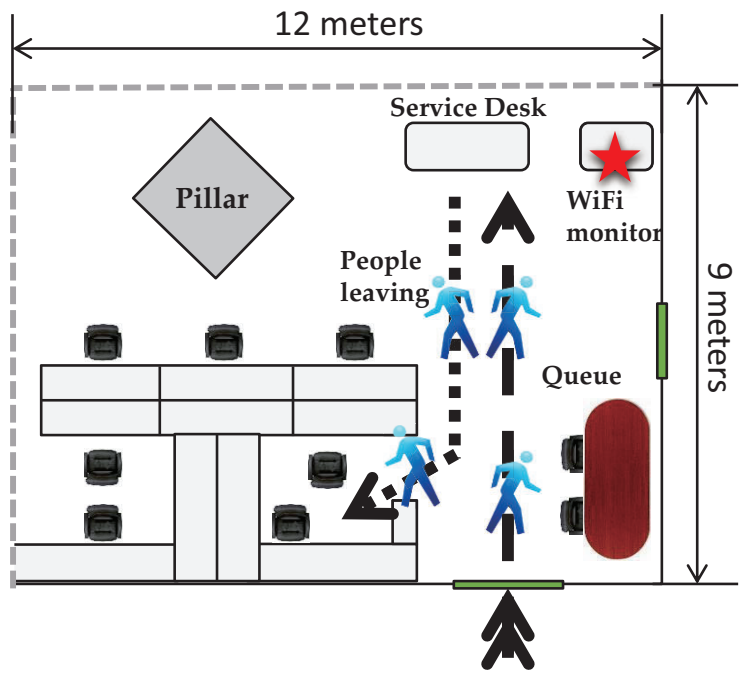

Figure 3: Illustration of experimental setup for queue measurement in the laboratory.

(BoS) in the queue following the temporal-reversed order. The LP/BoS Estimator takes the view point that the leaving point separates the service period and the leaving period, and thus the $\mathrm{WiFi}$ signals before LP is relatively stable whereas they drop dramatically after the leaving point. This useful observation indicates that the distributions of WiFi signal changes (i.e., slopes of RSS) before and after the leaving point are significantly different. This inspires us to identify LP by using the KL-divergence technique to examine the distribution difference of WiFi signal changes. And LP is determined as the time point (prior to EoL) separating the RSS slopes into two parts that present the most different distributions from each other.

In particular, we calculate the distributions of quantized RSS slopes before and after each time point $t_{j}$ occurring before EoL, respectively denoted as $P\left(K_{j-1}\right)$ and $Q\left(K_{j}\right), j=[1, \ldots, J]$, where $J$ is number of time points occurring before EoL. The K-L divergence between these two distributions are derived as:

$$
D_{K L}\left(P\left(K_{j-1}\right) \| Q\left(K_{j}\right)\right)=\sum_{q \in Q} P\left(K_{j-1}=q\right) \ln \frac{P\left(K_{j-1}=q\right)}{Q\left(K_{j}=q\right)},
$$

where $Q$ is the set of all possible values for quantized RSS slopes. $\mathrm{LP}$ is then determined as the time point $t_{j}$, which maximizes the K-L divergence value:

$$
t_{j}=\arg \max _{t_{j}}\left(D_{K L}\left(P\left(K_{j-1}\right) \| Q\left(K_{j}\right)\right)\right) .
$$

Similarly, our scheme can also employ the KL-divergence technique to identify BoS, since WiFi signals is relatively stable during the service period while they exhibit an obvious increasing trend in the waiting period.

\section{PERFORMANCE EVALUATION}

We next show our system implementation with the input RSS from single antenna as well as the maximum RSS from two antennas at the WiFi monitor, and evaluate their impacts.

Experimental Setup. We conduct experiments with human queues in a laboratory setup as shown in Figure 3. We place a WiFi monitor equipped with two antennas at the service desk to capture WiFi signals. We develop an Android app to send beacon packets at the rate

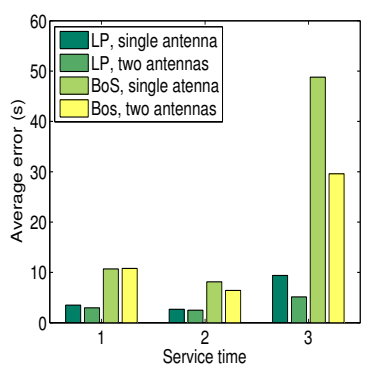

(a) LP \& BoS

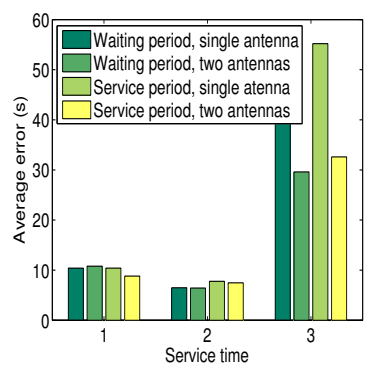

(b) Waiting \& service times
Figure 4: Performance comparison of the single-antenna and two-antenna approach under different service times in laboratory.

of $10 \mathrm{pkt} / \mathrm{sec}$. We experiment with three different service times, $30 s, 60 s$, and 180 s, representing short, normal and long service times, respectively. In total we collect 90 traces in the laboratory environment and we use two-fold cross-validation for evaluation. We calculate the estimation error of the relevant time points and important time periods with regard to the manually logged groundtruth.

Experimental Results. We first study the performance of the feature-driven scheme based on the signals received from a single and two antennas at the WiFi monitor. Figure 4 (a) compares the average error of estimating the LP and BoS under different length of the service time. We observe that the feature-driven scheme performs well with both single and two antennas under short (30s) and normal (60s) service times with the average error less than 10s. We find that with two antennas the system has better performance, especially under the long service time (180s). Moreover, Figure 10(b) depicts the average error of tracking the waiting and service times derived from the estimated BoS and LP. We observe the average error of both waiting and service times is less than 10s under short and normal service times. Under the long service time, using two antennas outperforms using single antenna for over $30 \%$, although both schemes experience performance degradation.

\section{REFERENCES}

[1] S. Isaacman and et.al., "Human mobility modeling at metropolitan scales," in ACM MobiSys, 2012.

[2] G. Chandrasekaran and et.al., "Vehicular speed estimation using received signal strength from mobile phones," in ACM Ubicomp, 2010.

[3] I. of Medicine (US), Hospital-Based Emergency Care: At the Breaking Point. National Academy Press, 2007.

[4] "Expo 2010 Shanghai China.” http://en.expo2010.cn/, 2010.

[5] U. Stilla, E. Michaelsen, U. Soergel, S. Hinz, and H. Ender, "Airborne monitoring of vehicle activity in urban areas," ISPRS, 2004.

[6] D. Bauer, M. Ray, and S. Seer, "Simple sensors used for measuring service times and counting pedestrians," Transportation Research Record, 2011.

[7] D. M. Bullock and et.al., "Automated measurement of wait times at airport security," Transportation Research Record, 2010.

[8] I. Siemens Industry, "Measure travel time and speed between multiple controller locations."

http://www.mobility.siemens.com, 2011. 